Article

\title{
Finite Series of Distributional Solutions for Certain Linear Differential Equations
}

\author{
Nipon Waiyaworn ${ }^{1}$, Kamsing Nonlaopon ${ }^{1, *(1)}$ and Somsak Orankitjaroen ${ }^{2}$ \\ 1 Department of Mathematics, Khon Kaen University, Khon Kaen 40002, Thailand; \\ nipon_waiyaworn@kkumail.com \\ 2 Department of Mathematics, Faculty of Science, Mahidol University, Bangkok 10400, Thailand; \\ somsak.ora@mahidol.ac.th \\ * Correspondence: nkamsi@kku.ac.th; Tel.: +66-8-6642-1582
}

Received: 31 August 2020; Accepted: 2 October 2020; Published: 13 October 2020

\begin{abstract}
In this paper, we present the distributional solutions of the modified spherical Bessel differential equations $t^{2} y^{\prime \prime}(t)+2 t y^{\prime}(t)-\left[t^{2}+v(v+1)\right] y(t)=0$ and the linear differential equations of the forms $t^{2} y^{\prime \prime}(t)+3 t y^{\prime}(t)-\left(t^{2}+v^{2}-1\right) y(t)=0$, where $v \in \mathbb{N} \cup\{0\}$ and $t \in \mathbb{R}$. We find that the distributional solutions, in the form of a finite series of the Dirac delta function and its derivatives, depend on the values of $v$. The results of several examples are also presented.
\end{abstract}

Keywords: Dirac delta function; distributional solution; Laplace transform; power series solution

\section{Introduction}

It is well known that the linear differential equation of the form

$$
\sum_{n=0}^{m} a_{n}(t) y^{(n)}(t)=0, \quad a_{m}(t) \neq 0,
$$

where $a_{n}(t)$ is an infinitely smooth coefficient for each $n$, and has no distributional solutions other than the classical ones. However, if the leading coefficient $a_{m}(t)$ has a zero, the classical solution of (1) may cease to exist in a neighborhood of that zero. In that case, (1) may have a distributional solution. It was not until 1982 that Wiener [1] proposed necessary and sufficient conditions for the existence of an Nth-order distributional solution to the differential equation (1). The Nth-order distributional solution that Wiener proposed is a finite sum of Dirac delta function and its derivatives:

$$
y(t)=\sum_{n=0}^{N} b_{n} \delta^{(n)}(t), \quad b_{N} \neq 0 .
$$

It can be easily verified by $(10)$ that $\delta(t)$ is a zero order distributional solution of the equation

$$
t y^{\prime \prime}(t)+2 y^{\prime}(t)+t y(t)=0 ;
$$

the Bessel equation

$$
t^{2} y^{\prime \prime}(t)+t y^{\prime}(t)+\left(t^{2}-1\right) y(t)=0 ;
$$

the confluent hypergeometric equation

$$
t y^{\prime \prime}(t)+(2-t) y^{\prime}(t)-y(t)=0 ;
$$


and the second order Cauchy-Euler equation

$$
t^{2} y^{\prime \prime}(t)+3 t y^{\prime}(t)+y(t)=0 .
$$

The distributional solutions with higher order of Cauchy-Euler equations were studied by many researchers; see [2-8] for more details.

The infinite order distributional solution of the form

$$
y(t)=\sum_{n=0}^{\infty} b_{n} \delta^{(n)}(t)
$$

to various differential equations in a normal form with singular coefficients was studied by many researchers [9-13]. Furthermore, a brief introduction to these concepts is presented by Kanwal [14].

In 1984, Cooke and Wiener [15] presented the existence theorems for distributional and analytic solutions of functional differential equations. In 1987, Littlejohn and Kanwal [16] studied the distributional solutions of the hypergeometric differential equation, whose solutions are in the form of (3). In 1990, Wiener and Cooke [17] presented the necessary and sufficient conditions for the simultaneous existence of solutions to linear ordinary differential equations in the forms of rational functions and (2).

As mentioned in abstract, we propose the distributional solutions of the modified spherical Bessel differential equations

$$
t^{2} y^{\prime \prime}(t)+2 t y^{\prime}(t)-\left[t^{2}+v(v+1)\right] y(t)=0
$$

and the linear differential equations of the forms

$$
t^{2} y^{\prime \prime}(t)+3 t y^{\prime}(t)-\left(t^{2}+v^{2}-1\right) y(t)=0,
$$

where $v \in \mathbb{N} \cup\{0\}$ and $t \in \mathbb{R}$. The modified spherical Bessel differential equation is just the spherical Bessel equation with a negative separation constant. The spherical Bessel equation occurs when dealing with the Helmholtz equation in spherical coordinates of various problems in physics such as a scattering problem [18].

We use the simple method, consisting of Laplace transforms of right-sided distributions and power series solution, for searching the distributional solutions of these equations. We find that the solutions are in the forms of finite linear combinations of the Dirac delta function and its derivatives depending on the values of $v$.

\section{Preliminaries}

In this section, we introduce the basic knowledge and concepts, which are essential for this work.

Definition 1. Let $\mathcal{D}$ be the space consisting of all real-valued functions $\varphi(t)$ with continuous derivatives of all orders and compact support. The support of $\varphi(t)$ is the closure of the set of all elements $t \in \mathbb{R}$ such that $\varphi(t) \neq 0$. Then $\varphi(t)$ is called a test function.

Definition 2. A distribution $T$ is a continuous linear functional on the space $\mathcal{D}$. The space of all such distributions is denoted by $\mathcal{D}^{\prime}$.

For every $T \in \mathcal{D}^{\prime}$ and $\varphi(t) \in \mathcal{D}$, the value that $T$ acts on $\varphi(t)$ is denoted by $\langle T, \varphi(t)\rangle$. Note that $\langle T, \varphi(t)\rangle \in \mathbb{R}$.

\section{Example 1.}

(i) The locally integrable function $f(t)$ is a distribution generated by the locally integrable function $f(t)$. Then we define $\langle f(t), \varphi(t)\rangle=\int_{\Omega} f(t) \varphi(t) d t$, where $\Omega$ is the support of $\varphi(t)$ and $\varphi(t) \in \mathcal{D}$. 
(ii) The Dirac delta function is a distribution defined by $\langle\delta(t), \varphi(t)\rangle=\varphi(0)$ and the support of $\delta(t)$ is $\{0\}$.

A distribution $T$ generated by a locally integrable function is called a regular distribution; otherwise, it is called a singular distribution.

Definition 3. The kth-order derivative of a distribution $T$, denoted by $T^{(k)}$, is defined by $\left\langle T^{(k)}, \varphi(t)\right\rangle=$ $(-1)^{k}\left\langle T, \varphi^{(k)}(t)\right\rangle$ for all $\varphi(t) \in \mathcal{D}$.

\section{Example 2.}

(i) $\left\langle\delta^{\prime}(t), \varphi(t)\right\rangle=-\left\langle\delta(t), \varphi^{\prime}(t)\right\rangle=-\varphi^{\prime}(0)$;

(ii) $\left\langle\delta^{(k)}(t), \varphi(t)\right\rangle=(-1)^{k}\left\langle\delta(t), \varphi^{(k)}(t)\right\rangle=(-1)^{k} \varphi^{(k)}(0)$.

Definition 4. Let $\alpha(t)$ be an infinitely differentiable function. We define the product of $\alpha(t)$ with any distribution $T$ in $\mathcal{D}^{\prime}$ by $\langle\alpha(t) T, \varphi(t)\rangle=\langle T, \alpha(t) \varphi(t)\rangle$ for all $\varphi(t) \in \mathcal{D}$.

Definition 5. If $y(t)$ is a singular distribution and satisfies the equation

$$
\sum_{m=0}^{n} a_{m}(t) y^{(n)}(t)=f(t),
$$

where $a_{m}(t)$ is an infinitely differentiable function and $f(t)$ is an arbitrary known distribution, in the sense of distribution, and is called a distributional solution of (4).

Definition 6. Let $M \in \mathbb{R}$ and $f(t)$ be a locally integrable function satisfying the following conditions:

(i) $f(t)=0$ for all $t<M$;

(ii) There exists a real number $c$ such that $e^{-c t} f(t)$ is absolutely integrable over $\mathbb{R}$.

The Laplace transform of $f(t)$ is defined by

$$
F(s)=\mathcal{L}\{f(t)\}=\int_{M}^{\infty} f(t) e^{-s t} d t
$$

where $s$ is a complex variable.

It is well known that if $f(t)$ is continuous, then $F(s)$ is an analytic function on the half-plane $\Re(s)>\sigma_{a}$, where $\sigma_{a}$ is an abscissa of absolute convergence for $\mathcal{L}\{f(t)\}$.

Recall that the Laplace transform $G(s)$ of a locally integrable function $g(t)$ satisfying the conditions of definition 6 , that is,

$$
G(s)=\mathcal{L}\{g(t)\}=\int_{M}^{\infty} g(t) e^{-s t} d t
$$

where $\Re(s)>\sigma_{a}$, can be written in the form $G(s)=\left\langle g(t), e^{-s t}\right\rangle$.

Definition 7. Let $S$ be the space of test functions of rapid decay containing the complex-valued functions $\phi(t)$ having the following properties:

(i) $\phi(t)$ is infinitely differentiable-i.e., $\phi(t) \in C^{\infty}(\mathbb{R})$;

(ii) $\phi(t)$, as well as its derivatives of all orders, vanish at infinity faster than the reciprocal of any polynomial which is expressed by the inequality

$$
\left|t^{p} \phi^{(k)}(t)\right|<C_{p k}
$$

where $C_{p k}$ is a constant depending on $p, k$, and $\phi(t)$. Then $\phi(t)$ is called a test function in the space $S$. 
Definition 8. A distribution of slow growth or tempered distribution $T$ is a continuous linear functional over the space $S$ of test function of rapid decay and contains the complex-valued functions-i.e., there is assigned a complex number $\langle T, \phi(t)\rangle$ with properties:

(i) $\left\langle T, c_{1} \phi_{1}(t)+c_{2} \phi_{2}(t)\right\rangle=c_{1}\left\langle T, \phi_{1}(t)\right\rangle+c_{2}\left\langle T, \phi_{2}(t)\right\rangle$ for $\phi_{1}(t), \phi_{2}(t) \in S$ and constants $c_{1}, c_{2} ;$

(ii) $\lim _{m \rightarrow \infty}\left\langle T, \phi_{m}(t)\right\rangle=0$ for every null sequence $\left\{\phi_{m}(t)\right\} \in S$.

We shall let $S^{\prime}$ denote the set of all distributions of slow growth.

Definition 9. Let $f(t)$ be a distribution satisfying the following properties:

(i) $\quad f(t)$ is a right-sided distribution, that is, $f(t) \in \mathcal{D}^{\prime}{ }_{R}$.

(ii) There exists a real number $c$ such that $e^{-c t} f(t)$ is a tempered distribution.

The Laplace transform of a right-sided distribution $f(t)$ satisfying (ii) is defined by

$$
F(s)=\mathcal{L}\{f(t)\}=\left\langle e^{-c t} f(t), X(t) e^{-(s-c) t}\right\rangle,
$$

where $X(t)$ is an infinitely differentiable function with support bounded on the left, which equals 1 over a neighbourhood of the support of $f(t)$.

For $\Re(s)>c$, the function $X(t) e^{-(s-c) t}$ is a testing function in the space $S$ and $e^{-c t} f(t)$ is in the space $S^{\prime}$. Equation (7) can be reduced to

$$
F(s)=\mathcal{L}\{f(t)\}=\left\langle f(t), e^{-s t}\right\rangle
$$

Now $F(s)$ is a function of $s$ defined over the right half-plane $\Re(s)>c$. Zemanian [19] proved that $F(s)$ is an analytic function in the region of convergence $\Re(s)>\sigma_{1}$, where $\sigma_{1}$ is the abscissa of convergence and $e^{-c t} f(t) \in S^{\prime}$ for some real number $c>\sigma_{1}$.

Example 3. Let $\delta(t)$ be the Dirac delta function, $H(t)$ be the Heaviside function, and $f(t)$ be a Laplace-transformable distribution in $\mathcal{D}^{\prime}{ }_{R}$. If $k$ is a positive integer, then the following holds:

(i) $\mathcal{L}\left\{\left(t^{k-1} H(t)\right) /(k-1) !\right\}=1 / s^{k}, \quad \Re(s)>0$;

(ii) $\mathcal{L}\{\delta(t)\}=1, \quad-\infty<\Re(s)<\infty$;

(iii) $\mathcal{L}\left\{\delta^{(k)}(t)\right\}=s^{k}, \quad-\infty<\Re(s)<\infty$;

(iv) $\mathcal{L}\left\{t^{k} f(t)\right\}=(-1)^{k} F^{(k)}(s), \quad \Re(s)>\sigma_{1}$;

(v) $\mathcal{L}\left\{f^{(k)}(t)\right\}=s^{k} F(s), \quad \Re(s)>\sigma_{1}$.

The proof of following lemma 1 is given in [14].

Lemma 1. Let $\psi(t)$ be an infinitely differentiable function. Then

$$
\begin{aligned}
\psi(t) \delta^{(m)}(t)= & (-1)^{m} \psi^{(m)}(0) \delta(t)+(-1)^{m-1} m \psi^{(m-1)}(0) \delta^{\prime}(t) \\
& +(-1)^{m-2} \frac{m(m-1)}{2 !} \psi^{(m-1)}(0) \delta^{\prime \prime}(t)+\cdots+\psi(0) \delta^{(m)}(t) .
\end{aligned}
$$

A useful formula that follows from (9), for any monomial $\psi(t)=t^{n}$, is that

$$
t^{n} \delta^{(m)}(t)= \begin{cases}0, & \text { if } m<n \\ (-1)^{n} \frac{m !}{(m-n) !} \delta^{(m-n)}(t), & \text { if } m \geq n\end{cases}
$$




\section{Main Results}

In this section, we will state our main results and give their proofs.

Theorem 1. Consider the differential equation of the form

$$
t^{2} y^{\prime \prime}(t)+2 t y^{\prime}(t)-\left[t^{2}+v(v+1)\right] y(t)=0,
$$

where $v \in \mathbb{N} \cup\{0\}$ and $t \in \mathbb{R}$. The distributional solutions of (11) are given by

$$
y(t)=P_{v}(D) \delta(t),
$$

where

$$
P_{v}(D)=\frac{1}{2^{v}} \sum_{k=0}^{\lfloor v / 2\rfloor}(-1)^{k} \frac{(2 v-2 k) !}{k !(v-k) !(v-2 k) !} D^{v-2 k},
$$

is a Legendre polynomial of distributional derivative operator $D=d / d t$.

Proof. Applying the Laplace transform to both sides of (11) with $\mathcal{L}\{y(t)\}=F(s)$, and using Example 3(iv), (v), we obtain

$$
\left(1-s^{2}\right) F^{\prime \prime}(s)-2 s F^{\prime}(s)+v(v+1) F(s)=0 .
$$

Suppose that a solution of (13) is of the form $F(s)=\sum_{n=0}^{\infty} a_{n} s^{n}$. Differentiating term by term, we obtain

$$
F^{\prime}(s)=\sum_{n=1}^{\infty} n a_{n} s^{n-1}
$$

and

$$
F^{\prime \prime}(s)=\sum_{n=2}^{\infty} n(n-1) a_{n} s^{n-2}
$$

Substituting these terms into (13), we have

$$
\begin{aligned}
& {\left[2 a_{2}+v(v+1) a_{0}\right]+\left[(3 \cdot 2) a_{3}-\left(2-v(v+1) a_{1}\right] s\right.} \\
& +\sum_{n=2}^{\infty}\left[(n+2)(n+1) a_{n+2}-n(n-1) a_{n}-2 n a_{n}+v(v+1) a_{n}\right] s^{n}=0 .
\end{aligned}
$$

Since $s^{n} \neq 0$, it follows that

$$
\begin{gathered}
2 a_{2}+v(v+1) a_{0}=0, \quad(3 \cdot 2) a_{3}-\left(2-v(v+1) a_{1}=0,\right. \\
(n+2)(n+1) a_{n+2}-n(n-1) a_{n}-2 n a_{n}+v(v+1) a_{n}=0, \quad n \geq 2,
\end{gathered}
$$

which leads to a recurrence relation

$$
a_{n+2}=-\frac{(v-n)(v+n+1)}{(n+1)(n+2)} a_{n} .
$$


Thus, we obtain

$$
\begin{aligned}
a_{2} & =-\frac{v(v+1)}{2 !} a_{0} \\
a_{4} & =(-1)^{2} \frac{v(v-2)(v+1)(v+3)}{4 !} a_{0} \\
& \vdots \\
a_{2 n} & =(-1)^{n} \frac{v(v-2) \cdots(v-2 n+2)(v+1)(v+3) \cdots(v+2 n-1)}{(2 n) !} a_{0} .
\end{aligned}
$$

Similarly,

$$
\begin{aligned}
a_{3} & =-\frac{(v-1)(v+2)}{2 \cdot 3} a_{1} \\
a_{5} & =(-1)^{2} \frac{(v-1)(v-3)(v+2)(v+4)}{5 !} a_{1} \\
& \vdots \\
a_{2 n+1} & =(-1)^{n} \frac{(v-1)(v-3) \cdots(v-2 n+1)(v+2)(v+4) \cdots(v+2 n)}{(2 n+1) !} a_{1} .
\end{aligned}
$$

Letting $a_{0}=a_{1}=1$, we get the two solutions of (13) in the forms

$$
F_{e}(s)=1+\sum_{n=1}^{\infty}(-1)^{n} \frac{v(v-2) \cdots(v-2 n+2)(v+1)(v+3) \cdots(v+2 n-1)}{(2 n) !} s^{2 n}
$$

and

$$
F_{o}(s)=s+\sum_{n=1}^{\infty}(-1)^{n} \frac{(v-1)(v-3) \cdots(v-2 n+1)(v+2)(v+4) \cdots(v+2 n)}{(2 n+1) !} s^{2 n+1} .
$$

If $v$ is even, letting $v=2 m, m \in \mathbb{N} \cup\{0\}$, we note that

$$
v(v-2) \cdots(v-2 n+2)=2 m(2 m-2) \cdots(2 m-2 n+2)= \begin{cases}0, & m=0 ; \\ \frac{2^{n} m !}{(m-n) !}, & m>0, n \leq m ; \\ 0, & n>m>0,\end{cases}
$$

and

$$
(v+1)(v+3) \cdots(v+2 n-1)=(2 m+1)(2 m+3) \cdots(2 m+2 n-1)=\frac{(2 m+2 n) ! m !}{2^{n}(2 m) !(m+n) !} .
$$

Then, in this case, $F_{e}(s)$ only becomes the finite series of the form

$$
F_{e}(s)=\frac{(m !)^{2}}{(2 m) !} \sum_{k=0}^{m}(-1)^{k} \frac{(2 m+2 k) ! s^{2 k}}{(m-k) !(m+k) !(2 k) !} .
$$

If $v$ is odd, letting $v=2 m+1, m \in \mathbb{N} \cup\{0\}$, we note that

$$
(v-1)(v-3) \cdots(v-2 n+1)=2 m(2 m-2) \cdots(2 m-2 n+2)= \begin{cases}0, & m=0 \\ \frac{2^{n} m !}{(m-n) !}, & m>0, n \leq m \\ 0, & n>m>0\end{cases}
$$


and

$$
(v+2)(v+4) \cdots(v+2 n)=(2 m+3)(2 m+5) \cdots(2 m+2 n+1)=\frac{(2 m+2 n+1) ! m !}{2^{n}(2 m+1) !(m+n) !} .
$$

Then, in this case, $F_{0}(s)$ only becomes the finite series of the form

$$
F_{o}(s)=\frac{(m !)^{2}}{(2 m+1) !} \sum_{k=0}^{m}(-1)^{k} \frac{(2 m+2 k+1) ! s^{2 k+1}}{(m-k) !(m+k) !(2 k+1) !} .
$$

For $v=0,1,2, \ldots$, we have $F_{v}(s)$, as follows:

$$
\begin{aligned}
& F_{0}(s)=1=P_{0}(s) \\
& F_{1}(s)=s=P_{1}(s) \\
& F_{2}(s)=1-3 s^{2}=-2 P_{2}(s) \\
& F_{3}(s)=s-(5 / 3) s^{3}=-(2 / 3) P_{3}(s), \\
& F_{4}(s)=1-10 s^{2}+(35 / 3) s^{4}=(8 / 3) P_{4}(s), \\
& F_{5}(s)=s-(14 / 3) s^{3}+(21 / 5) s^{5}=(8 / 15) P_{5}(s), \\
& F_{6}(s)=1-21 s^{2}+63 s^{4}-(231 / 5) s^{6}=-(16 / 5) P_{6}(s), \\
& F_{7}(s)=s-9 s^{3}+(99 / 5) s^{5}-(429 / 35) s^{7}=-(16 / 35) P_{7}(s),
\end{aligned}
$$

and so on, where $P_{n}(s)$ is the Legendre polynomial of $s$ for $n=0,1,2, \ldots$ Since (13) is linear, $P_{v}$ is also its solution for all non-negative integer $v$. Taking the inverse Laplace transform to $P_{v}(s)$, and using Example 3(ii),(iii), we obtain the solutions of (11),

$$
\begin{aligned}
y(t) & =\frac{1}{2^{v}} \sum_{k=0}^{\lfloor v / 2\rfloor}(-1)^{k} \frac{(2 v-2 k) !}{k !(v-k) !(v-2 k) !} D^{v-2 k} \delta(t), \quad\left(D \equiv \frac{d}{d t} \text { distribution derivative }\right) \\
& =P_{v}(D) \delta(t)
\end{aligned}
$$

which are the distributional solutions of the form (12).

Example 4. Letting $v=1$, (11) becomes

$$
t^{2} y^{\prime \prime}(t)+2 t y^{\prime}(t)-\left(t^{2}+2\right) y(t)=0 .
$$

From Theorem 1, (18) has a solution

$$
y(t)=\delta^{\prime}(t)
$$

Letting $v=4$, (11) becomes

$$
t^{2} y^{\prime \prime}(t)+2 t y^{\prime}(t)-\left(t^{2}+20\right) y(t)=0
$$

From Theorem 1, (20) has a solution

$$
y(t)=\frac{35}{8} \delta^{(4)}(t)-\frac{15}{4} \delta^{\prime \prime}(t)+\frac{3}{8} \delta(t) .
$$

By applying (10), it is not difficult to verify that (19) and (21) satisfy (18) and (20), respectively.

Theorem 2. Consider the equation of the form

$$
t^{2} y^{\prime \prime}(t)+3 t y^{\prime}(t)-\left(t^{2}+v^{2}-1\right) y(t)=0
$$


where $v \in \mathbb{N} \cup\{0\}$ and $t \in \mathbb{R}$. The distributional solutions of (22) are given by

$$
y(t)=T_{v}(D) \delta(t)
$$

where

$$
T_{v}(D)=\frac{v}{2} \sum_{k=0}^{\lfloor v / 2\rfloor}(-1)^{k} \frac{2^{v-2 k}(n-k-1) !}{k !(v-2 k) !} D^{v-2 k},
$$

is a Chebyshev polynomial of the first kind of distributional derivative operator $D=d / d t$.

Proof. Applying the Laplace transform $\mathcal{L}\{y(t)\}=F(s)$ to (22) and using Example 3(iv), (v), we obtain

$$
\left(1-s^{2}\right) F^{\prime \prime}(s)-s F^{\prime}(s)+v^{2} F(s)=0 .
$$

Suppose that a solution of (24) is of the form $F(s)=\sum_{n=0}^{\infty} a_{n} s^{n}$. Differentiating $F(s)$ term by term, we obtain

$$
F^{\prime}(s)=\sum_{n=1}^{\infty} n a_{n} s^{n-1}
$$

and

$$
F^{\prime \prime}(s)=\sum_{n=2}^{\infty} n(n-1) a_{n} s^{n-2}
$$

Substituting these terms into (24), we get

$$
2 a_{2}+v^{2} a_{0}+\left(6 a_{3}-a_{1}+v^{2} a_{1}\right) s+\sum_{n=2}^{\infty}\left\{(n+2)(n+1) a_{n+2}-\left[n(n-1)+n-v^{2}\right] a_{n}\right\} s^{n}=0 .
$$

Since $s^{n} \neq 0$, it follows that

$$
\begin{aligned}
2 a_{2}+v^{2} a_{0} & =0, \\
6 a_{3}-a_{1}+v^{2} a_{1} & =0,
\end{aligned}
$$

and for $n=2,3, \ldots$,

$$
(n+2)(n+1) a_{n+2}-\left(n^{2}-v^{2}\right) a_{n}=0 .
$$

Hence,

$$
\begin{aligned}
& a_{2}=-\frac{v^{2}}{2} a_{0}, \\
& a_{3}=\frac{1-v^{2}}{3 !} a_{1},
\end{aligned}
$$

and for $n=2,3, \ldots$,

$$
a_{n+2}=\frac{n^{2}-v^{2}}{(n+2)(n+1)} a_{n}
$$

Thus, we obtain

$$
\begin{aligned}
& a_{2}=-\frac{v^{2}}{2} a_{0}, \\
& a_{4}=\frac{\left(2^{2}-v^{2}\right)\left(-v^{2}\right)}{4 !} a_{0},
\end{aligned}
$$


and so on. Similarly,

$$
\begin{aligned}
& a_{3}=\frac{1-v^{2}}{3 !} a_{1}, \\
& a_{5}=\frac{\left(3^{2}-v^{2}\right)\left(1-v^{2}\right)}{5 !} a_{1}
\end{aligned}
$$

and so on. A pattern clearly emerges:

$$
a_{2 n}=\frac{\left[(2 n-2)^{2}-v^{2}\right]\left[(2 n-4)^{2}-v^{2}\right] \cdots\left(2^{2}-v^{2}\right)\left(-v^{2}\right)}{(2 n) !} a_{0}
$$

and

$$
a_{2 n+1}=\frac{\left[(2 n-1)^{2}-v^{2}\right]\left[(2 n-3)^{2}-v^{2}\right] \cdots\left(3^{2}-v^{2}\right)\left(1-v^{2}\right)}{(2 n+1) !} a_{1} .
$$

Hence, we get two solutions of (24) in the forms

$$
F_{e}(s)=a_{0}+\sum_{n=1}^{\infty} \frac{\left[(2 n-2)^{2}-v^{2}\right]\left[(2 n-4)^{2}-v^{2}\right] \cdots\left(2^{2}-v^{2}\right)\left(-v^{2}\right)}{(2 n) !} a_{0} s^{2 n}
$$

and

$$
F_{o}(s)=a_{1} s+\sum_{n=1}^{\infty} \frac{\left[(2 n-1)^{2}-v^{2}\right]\left[(2 n-3)^{2}-v^{2}\right] \cdots\left(3^{2}-v^{2}\right)\left(1-v^{2}\right)}{(2 n+1) !} a_{1} s^{2 n+1} .
$$

If $v$ is even, letting $v=2 m, m \in \mathbb{N} \cup\{0\}$, then $a_{2 m+2}=0$, so that $a_{2 n+2}=0$ for $n \geq m$. Hence,

$$
F_{e}(s)=1+\sum_{n=1}^{m} \frac{\left[(2 n-2)^{2}-(2 m)^{2}\right]\left[(2 n-4)^{2}-(2 m)^{2}\right] \cdots\left[2^{2}-(2 m)^{2}\right]\left[-(2 m)^{2}\right]}{(2 n) !} s^{2 n},
$$

equivalently to

$$
F_{e}(s)=1+\sum_{n=1}^{m} \frac{\prod_{k=1}^{n} 4(n-k+m)(n-k-m)}{(2 n) !} s^{2 n} .
$$

If $v$ is odd, letting $v=2 m+1, m \in \mathbb{N} \cup\{0\}$, then $a_{2 m+3}=0$, so that $a_{2 n+3}=0$ for $n \geq m$. Hence,

$$
F_{o}(s)=s+\sum_{n=1}^{m} \frac{\left.\left[(2 n-1)^{2}-(2 m+1)^{2}\right]\left[(2 n-3)^{2}-(2 m+1)^{2}\right] \cdots\left[3^{2}-(2 m+1)^{2}\right)\right]\left[1-(2 m+1)^{2}\right]}{(2 n+1) !} s^{2 n+1},
$$

equivalently to

$$
F_{o}(s)=s+\sum_{n=1}^{m} \frac{\prod_{k=1}^{n} 4(n-k+m+1)(n-k-m)}{(2 n+1) !} s^{2 n+1}
$$


For $v=0,1,2, \ldots$, we have $F_{v}(s)$, as follows:

$$
\begin{aligned}
& F_{0}(s)=1=T_{0}(s) \\
& F_{1}(s)=s=T_{1}(s) \\
& F_{2}(s)=1-2 s^{2}=-T_{2}(s) \\
& F_{3}(s)=s-(4 / 3) s^{3}=-(1 / 3) T_{3}(s) \\
& F_{4}(s)=1-8 s^{2}+8 s^{4}=T_{4}(s) \\
& F_{5}(s)=s-4 s^{3}+(16 / 5) s^{5}=(1 / 5) T_{5}(s), \\
& F_{6}(s)=1-18 s^{2}+48 s^{4}-32 s^{6}=-T_{6}(s), \\
& F_{7}(s)=s-8 s^{3}+16 s^{5}-(64 / 7) s^{7}=-(1 / 7) T_{7}(s),
\end{aligned}
$$

and so on, where $T_{n}(s)$ is the Chebyshev polynomial of the first kind of $s$ for $n=0,1,2, \ldots$ Since (24) is linear, $T_{n}(s)$ is also its solution. Taking the inverse Laplace transform to $T_{n}(s)$, and using Example 3(ii),(iii), we obtain the solutions of (22),

$$
\begin{aligned}
y_{n}(t) & =\frac{v}{2} \sum_{k=0}^{\lfloor v / 2\rfloor}(-1)^{k} \frac{2^{v-2 k}(n-k-1) !}{k !(v-2 k) !} D^{v-2 k} \delta(t), \\
& =T_{n}(D) \delta(t)
\end{aligned}
$$

which are the distributional solutions of the forms (23).

Example 5. Letting $v=1$, (22) becomes

$$
t^{2} y^{\prime \prime}(t)+3 t y^{\prime}(t)-t^{2} y(t)=0 .
$$

From Theorem 2, (27) has a solution

$$
y(t)=\delta^{\prime}(t)
$$

Letting $v=4$, (22) becomes

$$
t^{2} y^{\prime \prime}(t)+2 t y^{\prime}(t)-\left(t^{2}+15\right) y(t)=0 .
$$

From Theorem 2, (29) has a solution

$$
y(t)=8 \delta^{(4)}(t)-8 \delta^{\prime \prime}(t)+\delta(t) .
$$

By applying (10), it is not difficult to verify that (28) and (30) satisfy (27) and (29), respectively.

\section{Conclusions}

In this paper, we seek the distributional solutions of the modified spherical Bessel differential Equation (11) and the linear differential equation of the form (22) by using the Laplace transforms of right-sided distributions and the power series solutions. The obtained solutions in the forms of the finite linear combinations of the Dirac delta function and its derivatives depend on the value of $v$, to which their coefficients regard the coefficients of Legendre and Chebyshev polynomials (see [20] for more details). However, for solutions of (11) and (22) in the usual sense, not mentioned here, they can be seen in many standard and technical textbooks (see, for example, Ross [21]) but, even more, may appear in models related to equilibrium of membrane structures, steady states of evolutive equations or nonlinear science (see studies [22-25]). 
Author Contributions: All authors contributed equally to this article. They read and approved the final manuscript. All authors have read and agreed to the published version of the manuscript.

Funding: This research received no external funding.

Acknowledgments: This work was supported by the Research Fund for Supporting Lecturer to Admit High Potential Student to Study and Research on His Expert Program Year 2018 from the Graduate School, Khon Kaen University, Thailand (Grant no. 611T105).

Conflicts of Interest: The authors declare no conflict of interest.

\section{References}

1. Wiener, J. Generalized-function solutions of differential and functional differential equations. J. Math. Anal. Appl. 1982, 88, 170-182. [CrossRef]

2. Kananthai, A. Distribution solutions of the third order Euler equation. Southeast Asian Bull. Math. 1999, 23, 627-631.

3. Liangprom, A.; Nonlaopon, K. On the generalized solutions of a certain fourth order Euler equations. J. Nonlinear Sci. Appl. 2017, 10, 4077-4084. [CrossRef]

4. Sacorn, N.; Nonlaopon, K.; Kim, H. A note on the generalized solutions of the third-order Cauchy-Euler equations. Commun. Math. Appl. 2018, 9, 661-669.

5. Jodnok, P.; Nonlaopon, K. On the generalized solutions of the fifth order Euler equations. Far East J. Math. Sci. 2018, 106, 59-74. [CrossRef]

6. Mirumbe, G.I.; Mango, J.M. On generalized solutions of locally Fuchsian ordinary differential equations. J. Math. Sci. Adv. Appl. 2018, 51,99-117. [CrossRef]

7. Jhanthanam, S.; Nonlaopon, K.; Orankitjaroen, S. Generalized solutions of the third-order Cauchy-Euler equation in the space of right-sided distributions via Laplace transform. Mathematics 2019, 7, 376. [CrossRef]

8. Sangsuwan, A.; Nonlaopon, K.; Orankitjaroen, S.; Mirumbe, I. The generalized solutions of the $n$th order Cauchy-Euler equation. Mathematics 2019, 7, 932. [CrossRef]

9. Wiener, J. Generalized-function solutions of linear systems. J. Differ. Equ. 1980, 38, 301-315. [CrossRef]

10. Wiener, J.; Cooke, K.L.; Shah, S.M. Coexistence of analytic and distributional solutions for linear differential equations, II. J. Math. Anal. Appl. 1991, 159, 271-289. [CrossRef]

11. Hernandez, L.G.; Estrada, R. Solution of ordinary by series of delta functions. J. Math. Anal. Appl. 1995, 191, 40-55. [CrossRef]

12. Kanwal, R.P. Delta series solutions of differential and integral equations. Integral Transform. Spec. Funct. 1998, 6, 49-62. [CrossRef]

13. Nonlaopon, K.; Nuntigrangjana, T.; Putjuso, S. Distributional solutions of $n$ th-order differential equations of the Bessel equation. RMUTSB Acad. J. 2017, 5, 1-10.

14. Kanwal, R.P. Generalized Functions: Theory and Technique, 3rd ed.; Springer: New York, NY, USA, 2004.

15. Cooke, K.L.; Wiener, J. Distributional and analytic solutions of functional differential equations. J. Math. Anal. Appl. 1984, 98, 111-129. [CrossRef]

16. Littlejohn, L.L.; Kanwal, R.P. Distributional solutions of the hypergeometric differential equation. J. Math. Anal. Appl. 1987, 122, 325-345. [CrossRef]

17. Wiener, J.; Cooke, K.L. Coexistence of analytic and distributional solutions for linear differential equations, I. J. Math. Anal. Appl. 1990, 148, 390-421. [CrossRef]

18. Boyvel, L.P.; Jones, A.R. Electromagnetic Scattering and Its Applications; Applied Science Publishers: London, UK, 1981.

19. Zemanian, A.H. Distribution Theory and Transform Analysis; McGraw-Hill: New York, NY, USA, 1965.

20. Wiener, J. Generalized Solutions of Functional Differential Equations; World Scientific: Singapore, 1993.

21. Ross, S.L. Differential Equations, 3rd ed.; John Wiley \& Sons Inc.: Singapore, 1984.

22. Li, T.; Viglialoro, G. Analysis and explicit solvability of degenerate tensorial problems. Bound. Value Probl. 2018, 1, 1-13. [CrossRef]

23. Viglialoro, G.; Woolley, T.E. Boundedness in a parabolic-elliptic chemotaxis system with nonlinear diffusion and sensitivity and logistic source. Math. Methods Appl. Sci. 2018, 41, 1809-1824. [CrossRef] 
24. Az-Zóbi, E.A.; Al-Khaled, K.; Darweesh, A. Numeric-analytic solutions for nonlinear oscillators via the modified multi-stage decomposition method. Mathematics 2019, 7, 550. [CrossRef]

25. González-Gaxiola, O.; Santiago, J.A.; Ruiz de Chávez, J. Solution for the nonlinear relativistic harmonic oscillator via Laplace-Adomian decomposition method. Int. J. Appl. Comput. Math. 2017, 3, 2627-2638. [CrossRef]

(C) 2020 by the authors. Licensee MDPI, Basel, Switzerland. This article is an open access article distributed under the terms and conditions of the Creative Commons Attribution (CC BY) license (http://creativecommons.org/licenses/by/4.0/). 